\title{
Electrospun Scaffolds of Polylactide with a Different Enantiomeric Content and Loaded with Anti-Inflammatory and Antibacterial Drugs
}

\author{
Elena Llorens ${ }^{1}$, Luis J. del Valle ${ }^{*, 1,2}$, and Jordi Puiggalí ${ }^{1,2}$ \\ ${ }^{1}$ Departament d'Enginyeria Química, Universitat Politècnica de Catalunya, Av. Diagonal 647, Barcelona E-08028, Spain \\ ${ }^{2}$ Center for Research in Nano-Engineering (CrNE), Universitat Politècnica de Catalunya, Edifici C, \\ C/Pasqual i Vila s/n, Barcelona E-08028, Spain
}

Received February 9, 2015; Revised April 14, 2015; Accepted April 29, 2015

\begin{abstract}
Polylactide (PLA) electrospun microfibers were prepared and loaded with triclosan (TCS), ketoprofen (KTP), or their combination to obtain multifunctional scaffolds with bactericide and anti-inflammatory properties. Continuous and porous fibers with diameters in the micrometer scale and a unimodal distribution were successfully attained using a dual-electrospinning technique. Dual drug-loaded scaffolds showed a peculiar release that was in contrast to the single drug-loaded systems, which suggested the establishment of intermolecular interactions that delayed TCS and KTP release. Antimicrobial activity of all TCS-loaded electrospun scaffolds was demonstrated against E. coli and M. luteus bacteria; and furthermore, KTP-loaded samples slightly showed bactericide activity. Biocompatibility of scaffolds was evaluated by adhesion and proliferation assays, and interestingly, the dual drug-load systems were able to support high TCS doses without adverse effects.
\end{abstract}

Keywords: biomaterials, electrospinning, dual drug-release, polylactide, bactericide, triclosan, ketoprofen.

\section{Introduction}

Polylactide (PLA) is currently one of most attractive biodegradable polymers because it is derived from renewable resources, can be easily processed and has many applications ranging from commodity to specialty uses..$^{1-5}$ PLA is gaining special attention in the biomedical field. Specifically, devices including degradable sutures, drug releasing micro/nanoparticles, and porous scaffolds for cellular applications can be mentioned..$^{6-10}$ The great advantages of PLA are due in part to its ability to degrade into the naturally occurring metabolite lactic acid under physiological conditions, but other exceptional qualities, like biocompatibility, FDA approval for clinical use, low immunogenicity and good mechanical properties must also be considered.

PLA can be obtained by ring opening polymerization of L-lactide and/or D-lactide, a process that allows obtaining a large number of stereo-copolymers by changing their L/D ratio. Properties of PLA are strongly dependent on its optical purity; for example, PLA can range from semicrystalline to completely amorphous. ${ }^{11}$ The degree of crystallinity and even molecular orientation also has a strong influence on PLA biodegradability; specifically, the selection of adequate processing conditions is important to achieve a specific orientation for a given stereoregularity. For example, Lee et al. concluded

*Corresponding Author. E-mail: luis.javier.del.valle@upc.edu that molecular orientation in amorphous PLA increased with decreasing stretching temperature whereas semicrystalline samples required increasing temperatures. ${ }^{12}$ It seems that, for amorphous samples, the most important effect concerns the highest possible orientation when molecular relaxation is hindered by a temperature decrease. For semicrystalline samples, the orientation phenomenon involves the deformation of formed spherulites, and consequently higher temperatures are needed.

Electrospinning is currently one of the simplest methods to draw nano and microfibers from a polymer solution. Basically, the surface tension of a liquid droplet can be counteracted by the electrostatic repulsion due to the charging of the body of the liquid by a sufficiently high voltage. The droplet is stretched and may form a liquid jet if its molecular cohesion is sufficiently high. ${ }^{13}$ Scaffolds prepared by electrospinning showed particularly good potential for drug delivery applications due to their large surface area and interconnected pore structure. ${ }^{14,15}$ Moreover, drug release behavior can be easily controlled by the morphology and composition of fibers. ${ }^{16,17}$ Drug-loaded electrospun scaffolds can also be easily fabricated into various shapes (e.g. membranes or tubes) for different applications, such as wound dressing and nerve conduits. ${ }^{18,19}$ To date, most studies of electrospun scaffolds for drug delivery have been focused on sustained release of a single drug. However, it is clear that the use of different drugs is a promising strategy that allows combination therapy and may even induce 
synergic effects. Studies on dual drug-loaded systems, mainly fibers fabricated by coaxial electrospinning ${ }^{20}$ and multilayered electrospun polymer mats, ${ }^{21}$ demonstrated different release kinetics from simple systems and/or that the severe initial burst release could be retarded or even negated.

One of the main applications for electrospun scaffolds is its potential use as a matrix for wound healing. The success in wound healing can be handled by the control of various events, such as accelerating tissue regeneration (e.g. by promoting cell proliferation), preventing rejection of the implanted material (e.g. by improving cell adhesion on the material or by locally suppressing the immune response), or reducing the risk of adverse effects associated with tissue damage (e.g. by preventing infection by opportunistic microorganisms). Thus, the release of two or more different drugs at the proper time and in appropriate doses may be required during treatment of wound healing. For example, post-surgical infection is a common problem after surgical procedures. This requires the release of some drugs, including antibiotics, from the drug-loaded biomaterial in a sustained manner to prevent infections over a short or long period. The rapid release of anti-inflammatory agents and analgesics is also necessary to reduce the cellular immune response and patient's pain, respectively. Thus, it is highly desirable and challenging to develop novel drug delivery carriers that can control the release of multiple drugs with different release kinetics.

In the present study, the feasibility of loading active agents of different nature and with diverse therapeutic effects into polylactide fibers is assessed with the aim of developing multifunctional electrospun materials for regenerative medicine applications. A system involving two drugs, triclosan (TCS) as the antibacterial agent and ketoprofen (KTP) as a
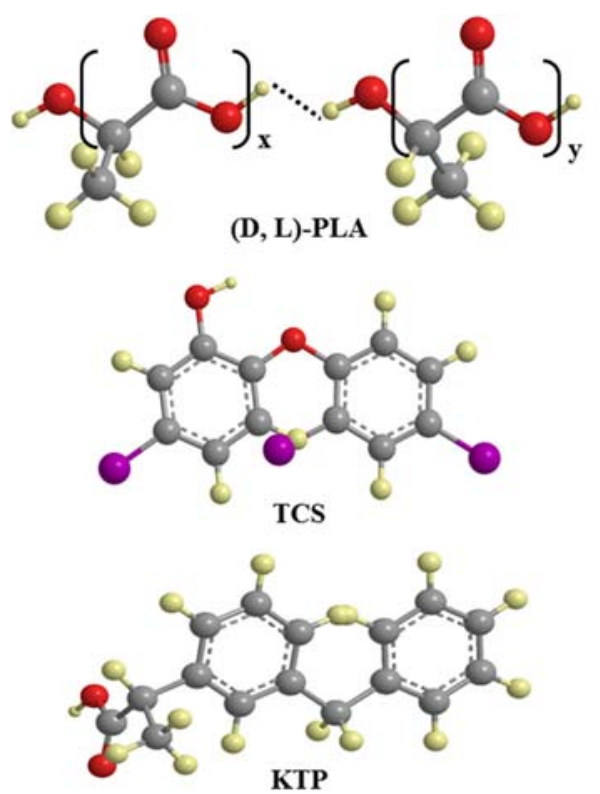

Figure 1. Chemical structures of polylactide (D,L-PLA), triclosan (TCS), and ketoprofen (KTP). conventional anti-inflammatory drug, was considered. Figure 1 shows the chemical structure of compounds used. Modulation of the therapeutic effect is expected to occur because of the difference in release of these drugs from scaffolds constituted by electrospun fibers. The influence of polymer matrix crystallinity on the release behavior is also determined. To this end, electrospun scaffolds were prepared using PLAs having slightly different ratios between L- and D-lactide units.

\section{Experimental}

Materials. Two commercial PLA grades from Natureworks ${ }^{\circledR}$ with 95.8 and $98 \mathrm{wt} \%$ L-lactic isomer content were used, i.e. PLA 2002D and PLA 4032D, respectively. According to the manufacturer, PLA 2002D is a transparent solid with a density of $1.24 \mathrm{~g} \cdot \mathrm{cc}^{-1}$. Its calorimetric and mechanical properties are defined by a glass transition temperature $\left(T_{g}\right)$ of $58{ }^{\circ} \mathrm{C}$, a melting point $\left(T_{m}\right)$ of $153{ }^{\circ} \mathrm{C}$, a tensile modulus $(E)$ of 3,500 MPa, a tensile strength $(\sigma)$ of 53-60 MPa and a tensile elongation $(\gamma)$ of $6 \%$. PLA 4032D has a translucent appearance, a density of $1.24 \mathrm{~g} \cdot \mathrm{cc}^{-1}$ and very different properties from the sample with higher D-lactide content. Thus, $T_{g}, T_{m}$, $E$, $\sigma$, and $\gamma$ values were reported to be $70^{\circ} \mathrm{C}, 160^{\circ} \mathrm{C}, 3,440$ 3,784 MPa, $103.2-144.5 \mathrm{MPa}$, and $100-180 \%$, respectively. Molecular weights determined by GPC were quite similar; specifically $M_{n}, M_{w}$, and polydispersity index were 98,100 $\mathrm{g} \cdot \mathrm{mol}^{-1}, 181,000 \mathrm{~g} \cdot \mathrm{mol}^{-1}$, and 1.85 for PLA 2002D and 84,200 $\mathrm{g} \cdot \mathrm{mol}^{-1}, 179,400 \mathrm{~g} \cdot \mathrm{mol}^{-1}$, and 2.13 for PLA 4032D. ${ }^{22,23}$

Trichloromethane (Chloroform), acetone, dimethylsulfoxide (DMSO), triclosan or irgasan (TCS), ketoprofen (KTP) and 3-(4,5-dimethylthiazol-2-yl)-2,5-diphenyl-2H-tetrazolium bromide (MTT) were purchased from Sigma-Aldrich (St. Louis, USA).

Vero (kidney epithelial cells extracted from African green monkey) and MDCK (kidney epithelial cells derived from Madin-Darby Canine) were purchased from ATCC (USA). Escherichia coli CECT 101 and Micrococcus luteus CECT 245 bacteria strains were obtained from the Spanish Collection of Type Culture (Valencia, Spain).

Drug-Loaded PLA Electrospun Microfibers. Scaffolds constituted by PLA 2002D and PLA 4032D microfibers were prepared by electrospinning, which also allowed loading the two selected drugs (i.e., TCS and KTP). The procedure consisted in dissolving the PLA in a chloroform-acetone mixture $(2: 1 v / v)$. For drug-loaded samples, $1 \mathrm{~mL}$ of dimethysulfoxide (DMSO) containing the corresponding drug was subsequently added and the mixture was vortexed to obtain an electrospinable solution. Final PLA concentration was $10 \%(\mathrm{w} / \mathrm{v})$ whereas for drug-loaded samples KTP and TCS concentrations were $1 \%(\mathrm{w} / \mathrm{v})$ and $3 \%(\mathrm{w} / \mathrm{v})$, respectively. A plastic syringe of $5 \mathrm{~mL}$ (Becton Dickson, Spain) was filled with the corresponding solution and electrospinning was carried out between the tip of a $18 \mathrm{G}$ needle (Terumo, Belgium) connected to the anode and the static collector connected to the cathode. 
The DC voltage was applied using a high voltage supply (ES30-5W model, Gamma High Voltage Research, USA). The flow rate was controlled using a KD100 infusion syringe pump (KD Scientific Inc., USA). The polymer jet was collected on an aluminum foil and $14 \mathrm{~mm}$ diameter coverslips to evaluate cell adhesion and proliferation. All electrospinning procedures were conducted at room temperature.

Morphology and Crystallinity of Dual Drug-Loaded PLA Microfibers. The diameter measurements and inspection of fiber textures were performed by scanning electron microscopy (SEM) using a Focus Ion Beam Zeiss Neon 40 instrument (Carl Zeiss, Germany). Carbon coating was accomplished with a Mitec K950 Sputter Coater (fitted with a film thickness monitor $k 150 \mathrm{x})$. Samples were viewed at an accelerating voltage of $5 \mathrm{kV}$.

Calorimetric data were obtained by differential scanning calorimetry (DSC) with a TA Instruments Q100 series equipped with a refrigeration cooling system (RCS). Experiments were conducted under a flow of dry nitrogen with a sample weight of approximately $5 \mathrm{mg}$ and calibration was performed with indium. Heating and cooling runs were carried out at a rate of $20^{\circ} \mathrm{C} \cdot \mathrm{min}^{-1}$ and $10^{\circ} \mathrm{C} \cdot \mathrm{min}^{-1}$, respectively.

Fourier transform infrared (FTIR) spectra were recorded on a FTIR 4100 Jasco spectrophotometer. The samples were placed in an attenuated total reflectance accessory with thermal control and a diamond crystal (Specac MKII Golden Gate Heated Single Reflection Diamond ATR).

Release Experiments. Controlled release experiments were performed with electrospun scaffolds cut into small square pieces $\left(20 \times 20 \times 0.1 \mathrm{~mm}^{3}\right)$. The samples were weighed and placed into polypropylene tubes. Two release media with different hydrophobicity and solvent ability were used: a typical phosphate buffer saline or (PBS) and PBS supplemented with 70\% (v/v) of ethanol. The second one was less hydrophilic than PBS and was interesting for simulating the supplement of serum in the medium. ${ }^{24}$ Drug release was carried out in $50 \mathrm{~mL}$ of the release medium at $25^{\circ} \mathrm{C}$ for 1 week. Drug concentration in the release medium was evaluated by UV-Vis spectroscopy using a UV-3600 spectrophotometer (Shimadzu, Japan). Calibration curves were obtained by plotting the absorbance measured at the corresponding wavelengths against drug concentration. Samples $(1 \mathrm{~mL})$ were drawn from the release medium at predetermined intervals and an equal volume of fresh medium was added to the release vessel. All drug release tests were carried out using three replicates to control the homogeneity of the release, and the results obtained were averaged.

Antimicrobial Test: Bacterial Growth and Adhesion Assays. Escherichia coli (E. coli) and Micrococcus luteus (M. luteus) bacteria were selected to evaluate the antimicrobial effect of TCS and KTP loaded electrospun scaffolds. The bacteria were previously grown aerobically to exponential phase in broth culture $\left(5 \mathrm{~g} \cdot \mathrm{L}^{-1}\right.$ beef extract, $5 \mathrm{~g} \cdot \mathrm{L}^{-1} \mathrm{NaCl}, 10 \mathrm{~g} \cdot \mathrm{L}^{-1}$ peptone, $\mathrm{pH}$ 7.2).

Growth experiments were performed in tubes of $15 \mathrm{~mL}$.
$10^{3} \mathrm{CFU}$ (colony forming units) were seeded in $5 \mathrm{~mL}$ of broth culture in the presence of the electrospun scaffolds. The cultures were incubated at $37^{\circ} \mathrm{C}$ and agitated at $80 \mathrm{rpm}$. After incubation for 24 and $48 \mathrm{~h}$, an aliquot of $100 \mu \mathrm{L}$ was diluted 10 times with distilled water and the absorbance at $600 \mathrm{~nm}$ was measured. The bacterial number was determined using a McFarland curve scale and graphically represented as relative percentages of the control.

Adhesion experiments were carried out by seeding $10^{7}-10^{8}$ CFU in $5 \mathrm{~mL}$ of broth culture in the presence of the electrospun scaffolds. The cultures were incubated at $37^{\circ} \mathrm{C}$ and agitated at $80 \mathrm{rpm}$. After incubation, the cultures were aspired and the material washed once with distilled water. Then, $1 \mathrm{~mL}$ of sterile $0.01 \mathrm{M}$ sodium thiosulfate was added and the mixture was vortexed for $2 \mathrm{~min}$ and left to repose for $30 \mathrm{~min}$ in order to remove the bacteria. Finally, $4 \mathrm{~mL}$ of broth culture was added and the tubes were incubated at $37^{\circ} \mathrm{C}$ for $24 \mathrm{~h}$ and agitated at $80 \mathrm{rpm}$. The bacterial number was determined as above indicated.

All assays were performed in triplicate and the results averaged. Analysis of variance (ANOVA) followed by Tukey test were conducted as statistical analyses at a confidence level of $95 \%(p<0.05)$. Additionally, correlation coefficients $(r)$ were calculated to evaluate the relation between antibacterial effect and drug release.

In vitro Biocompatibility Assays: Cell Adhesion and Proliferation. MDCK and Vero cells were cultured in Dulbecco's Modified Eagle Medium (DMEM) supplemented with $10 \%$ fetal bovine serum, $1 \%$ penicillin/streptomycin and $2 \mathrm{mM} \mathrm{L}$-glutamine at $37^{\circ} \mathrm{C}$ in a humidified atmosphere with $5 \% \mathrm{CO}_{2}$ and $95 \%$ air. The culture medium was changed every two days and, for sub-culture, cell monolayers were rinsed with phosphate buffered saline (PBS) and detached by incubation with trypsin-ethylenediaminetetraacetic acid (EDTA) $(0.25 \%)$ at $37^{\circ} \mathrm{C}$ for $2-5 \mathrm{~min}$. Cell concentration was determined by counting with a Neubauer chamber using $4 \%$ trypan-blue as dye vital. Detached cells with viability $\geq 95 \%$ were used for biocompatibility assays.

The unloaded and antioxidant loaded PLA electrospun microfibers were cut into square pieces $\left(10 \times 10 \times 0.1 \mathrm{~mm}^{3}\right)$. These samples were placed into the wells of a multi-well culture plate and sterilized by UV-radiation in a laminar flux cabinet for $15 \mathrm{~min}$. To fix the samples in the well, a small drop of silicone (Silbione ${ }^{\circledR}$ MED ADH 4300 RTV, Bluestar Silicones France SAS, Lyon, France) was used as the adhesive. Samples were incubated in $1 \mathrm{~mL}$ of the culture medium under culture conditions for $30 \mathrm{~min}$ to equilibrate the material. Finally, the medium was aspired and the material was evaluated for cell adhesion and proliferation by exposing cells to direct contact with the material surface.

To assess cell adhesion, aliquots of 50-100 $\mu \mathrm{L}$ containing $5 \times 10^{4}$ cells were seeded onto the electrospun scaffolds in the wells. The plate was incubated in culture conditions for 30 min to allow cell attachment to the material surface. Then, 
$1 \mathrm{~mL}$ of the culture medium was added to each well and the plate was incubated for $24 \mathrm{~h}$. Finally, cell viability was determined by the MTT (3-(4,5-dimethylthiazole-2-yl)-2,5-diphenyltetrazolium bromide) assay. Controls were performed by cell culture on the polystyrene surface of the plate (TCPS).

Cell proliferation was evaluated by a similar procedure to the adhesion assay, but the aliquot of 50-100 $\mu \mathrm{L}$ contained $2 \times 10^{4}$ cells. The cultures were maintained for 7 days to allow cell growth and adequate cell confluence in the well. The medium was renewed every two days. Finally, cell viability was determined by the MTT assay.

Each sample was evaluated using five replicates and the results were averaged and graphically represented. The statistical analysis was performed by one-way ANOVA to compare the means of all groups; Tukey test was then applied to determine a statistically significant difference between two studied groups. The tests were performed with a confidence level of $95 \%(p<0.05)$.

\section{Results and Discussion}

Morphology of Dual Drug-Loaded PLA Microfibers. Morphology of electrospun fibers depends on both solution properties (e.g. viscosity, surface tension, dielectric constant, volatility and concentration) and operational parameters (e.g. strength of the applied electrical field, tip-collector distance and flow rate). ${ }^{25-27}$ Selection of an appropriate solvent system is one of the most crucial points, especially in electrospinning of compounds with highly different characteristics (e.g. polymer and drugs). It should be pointed out that physicochemical properties of the solution can be affected by the incorporation of active drugs and that the system must be accurately chosen to support the load of one or both drugs and render appropriate electrospun fibers. ${ }^{28}$

It was reported that the selection of solvent was also crucial to control the diameter of PLA electrospun fibers. Thus, diameters could be easily varied from $100 \mathrm{~nm}$ (i.e., nanometer scale) to $2 \mu \mathrm{m}$ (i.e., micrometer scale) by using dimethyl formamide and chloroform-acetone $(2: 1 v / v)$ mixture as solvents, respectively, when polymer concentration was kept at $10 \%(\mathrm{w} / \mathrm{v}){ }^{29,30}$ Nevertheless, PLA concentration also played a determinant role because nanofibers could again be obtained when it was lower than $5 \%(\mathrm{w} / \mathrm{v}){ }^{31}$

The above conclusions were taken into account in this study since a micrometer scale was preferred to detect release differences between drugs and from distinct PLA matrices. The main problem was to choose a solvent based on the chloroform-acetone mixture able to dissolve $10 \%(\mathrm{w} / \mathrm{v})$ of PLA and the two selected drugs with solubility characteristics different from those of PLA. Note the large difference between Hildebrand parameters reported for PLA ${ }^{32}\left(\right.$ i.e., $\left.9.87\left(\mathrm{cal}^{-} \mathrm{cm}^{-3}\right)^{0.5}\right)$ and $\operatorname{TCS}^{33}\left(\right.$ i.e., $\left.14.38\left(\mathrm{cal}^{\cdot} \mathrm{cm}^{-3}\right)^{0.5}\right)$. Finally, drugs were dissolved in dimethyl sulfoxide and subsequently added to the former PLA solution to obtain a tricomponent solvent mixture
(chloroform:acetone:DMSO 6:3:1 $v / v / v$ ) compatible with the selected concentrations of PLA $(10 \%(\mathrm{w} / \mathrm{v}))$, TCS $(3 \%(\mathrm{w} / \mathrm{v}))$ and $\operatorname{KTP}(1 \%(\mathrm{w} / \mathrm{v}))$.

Figure 2 shows the SEM images of the fiber matrices and the corresponding diameter distribution analysis of electrospun fibers prepared under previously optimized operational parameters (i.e., applied voltage, flow rate and tip-collector distance of $15 \mathrm{kV}, 10 \mathrm{~mL} \cdot \mathrm{h}^{-1}$ and $12.5 \mathrm{~cm}$, respectively). A unimodal Gaussian diameter distribution was always determined (Figure 2, insets).

Average diameters determined for all samples are summarized in Table I. The success of the electrospinning procedure to obtain fibers in the micrometer scale is confirmed in all cases. Differences between unloaded fibers prepared from the two PLA grades were not highly significant (average value of $1.76 \mu \mathrm{m})$. However, the incorporation of the drug always led to a diameter increase following the order TCS/KTP $<$ TCS $<\mathrm{KTP}$, with average diameters being $1.98,2.82$, and $3.33 \mu \mathrm{m}$, respectively. Probably, the physicochemical characteristics of the electrospun solutions were more influenced by the incorporation of bigger KTP molecules (Figure 1). These caused the largest increase on the fiber diameter despite the drug load percentage was the smallest. It should also be pointed out that TCS and KTP drugs caused a significant discrepancy between fiber diameters of samples coming from the two PLA grades, with a higher increase being detected for PLA 4032D samples. These changes may reflect a different degree of molecular orientation and even different compactness of the amorphous phases between the two electrospun polymers. Finally, when the drugs, TCS and KTP, were loaded simultaneously in both PLA samples, diameter values were only slightly higher than those obtained for unloaded fibers (e.g. $1.77 \mu \mathrm{m}$ with respect to $1.66 \mu \mathrm{m}$ for the PLA 4032D, and $2.19 \mu \mathrm{m}$ regarding 1.86 for the PLA 2002D samples). This feature suggests some kind of interaction between TCS and KTP (e.g. hydrogen bonds between hydroxyl and carbonyl groups or stacking between aromatic groups) that could also be favored by the higher total drug content with respect to the single drug-loaded systems.

Figure 3 shows high-magnification SEM images of the different fibers which reveal a very similar porous texture for all cases.

Incorporation of Drugs into Electrospun Microfibers. FTIR spectra of unloaded and drug-loaded PLA 2002D samples in Figure 4 illustrate two interesting points. First, no significant differences exist between typical PLA absorption bands of scaffolds prepared from the two PLA grades or scaffolds loaded with the drugs or their combination. The given spectral region is sensitive to molecular conformation; consequently, it can be concluded that differences between electrospun samples were not large enough to be detected by FTIR. As will be discussed later, although the amorphous content may change slightly with the PLA grade and drug load, it seems that polymer chains attained a similar molecular conforma- 

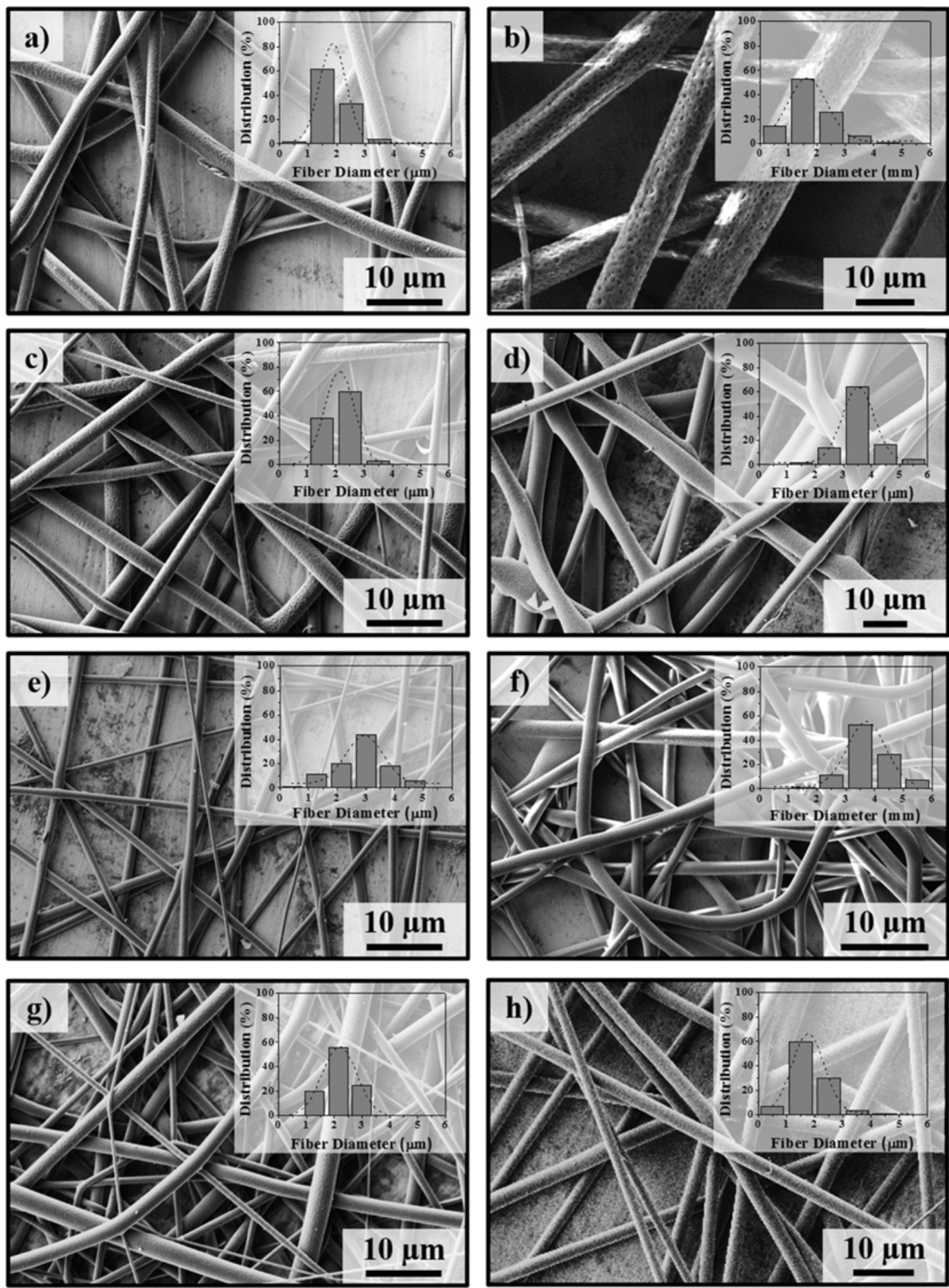

Figure 2. SEM micrographs of unloaded (a,b), triclosan-loaded (c,d), ketoprofen-loaded (e,f) and dual drug-loaded (g,h) in PLA 2002D (a,c,e,g) and PLA 4032D (b,d,f,h) electrospun microfibers.

tion after the electrospinning process. In fact, processed PLA microfibers were always practically amorphous but a high degree of molecular orientation was induced during electrospinning. ${ }^{34}$ Note, for example, that all spectra have similar intensity for the shoulder band at $1209 \mathrm{~cm}^{-1}\left(v_{\mathrm{as}}(\mathrm{C}-\mathrm{O}-\mathrm{C})+\right.$ $r_{\text {as }}\left(\mathrm{CH}_{3}\right)$ for a $10_{7}$ helical conformation) and the band at 1263 $\mathrm{cm}^{-1}(v(\mathrm{CH})+v(\mathrm{C}-\mathrm{O}-\mathrm{C})$ for a random conformation $)$. Also, the relative proportion between bands at $1387 \mathrm{~cm}^{-1}\left(\delta_{\mathrm{s}}\left(\mathrm{CH}_{3}\right)\right.$, amorphous form) and $1360 \mathrm{~cm}^{-1}(\delta(\mathrm{CH})$, semicrystalline form) became practically invariable. ${ }^{35}$ 
Table I. Diameters of Unloaded and Drug Loaded Electrospun PLA Fibers

\begin{tabular}{cc}
\hline Sample & Diameter $^{a, b}(\mu \mathrm{m})$ \\
\hline PLA 2002D & $1.86 \pm 0.06$ \\
PLA 4032D & $1.66 \pm 0.05$ \\
PLA 2002D + TCS & $2.12 \pm 0.01$ \\
PLA 4032D + TCS & $3.53 \pm 0.04$ \\
PLA 2002D + KTP & $2.95 \pm 0.02$ \\
PLA 4032D + KTP & $3.72 \pm 0.06$ \\
PLA 2002D + TCS/KTP & $2.19 \pm 0.01$ \\
PLA 4032D + TCS/KTP & $1.77 \pm 0.03$ \\
\hline
\end{tabular}

${ }^{a}$ Fibers were obtained using the same electrospinning operational parameters: applied voltage of $15 \mathrm{kV}$, flow rate of $10 \mathrm{~mL} / \mathrm{h}$ and tipcollector distance of $12.5 \mathrm{~cm} .{ }^{b}$ Mean value \pm standard deviation.
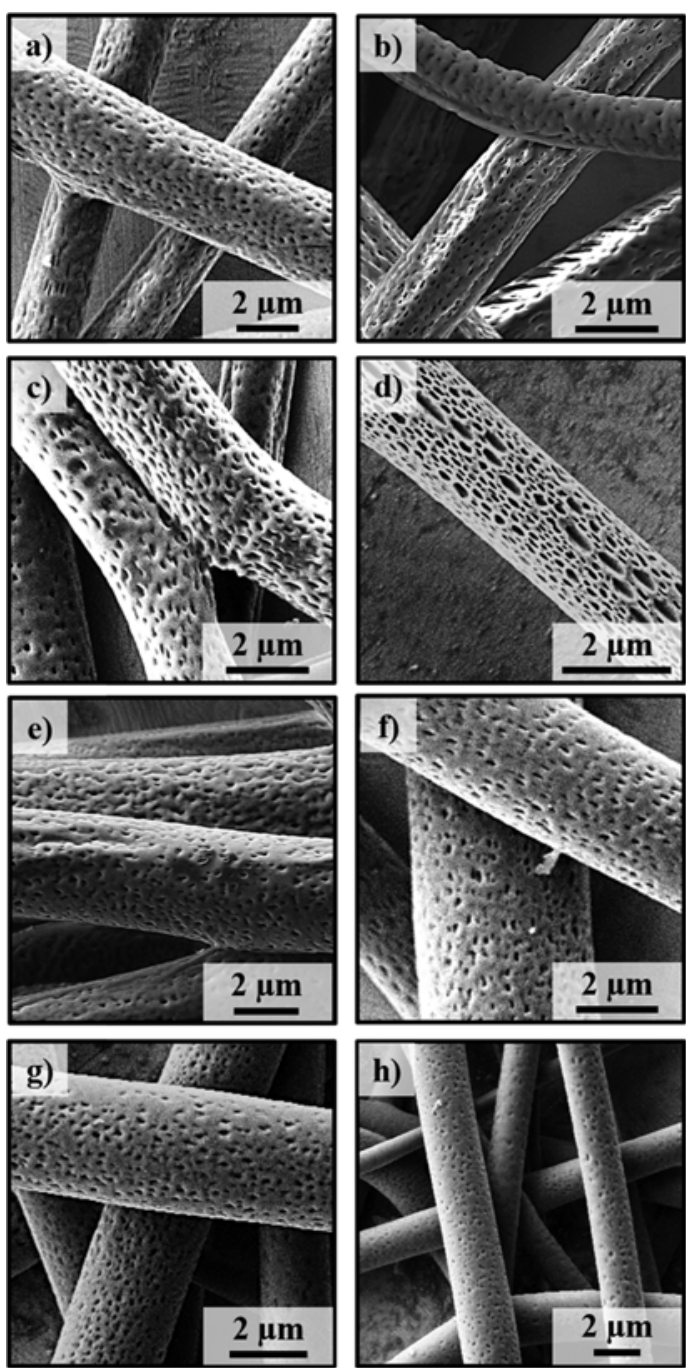

Figure 3. High-magnification SEM micrographs showing the surface texture of unloaded $(a, b)$, triclosan-loaded $(c, d)$, ketoprofen-loaded (e,f), and dual drug-loaded (g,h) PLA 2002D (a,c,e,g) and PLA 4032D (b,d,f,h) electrospun microfibers.

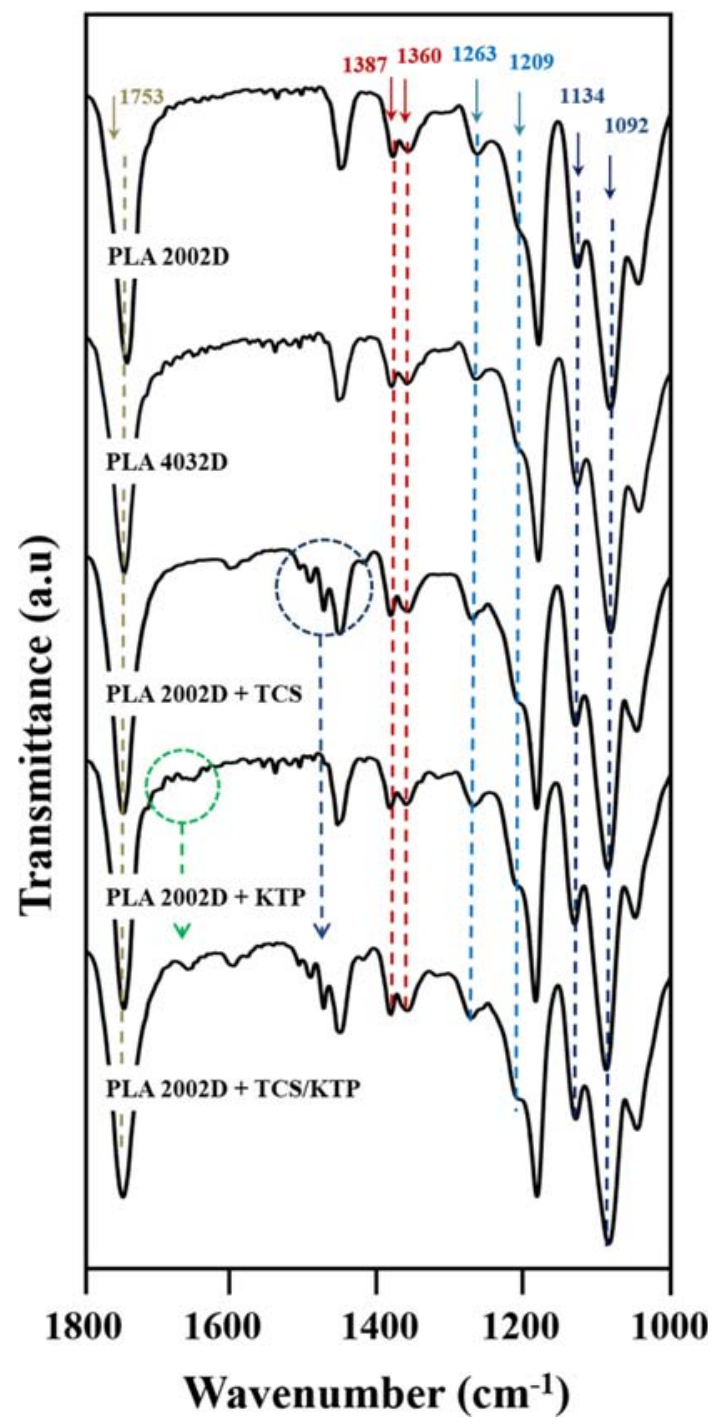

Figure 4. FTIR spectra (1800-1000 $\mathrm{cm}^{-1}$ region) of PLA 2002D, PLA 4032D, PLA 2002D + TCS, PLA 2002D + KTP, and PLA 2002D + TCS/KTP scaffolds.

FTIR spectra were sufficiently sensitive to detect the incorporated drugs despite the relatively low loading percentages. More precisely, TCS was clearly identified in the microfibers through the characteristic peak at $1476 \mathrm{~cm}^{-1}$, attributed to the $\mathrm{C}=\mathrm{C}$ stretching vibration of benzene rings. ${ }^{36}$ However, lesser loaded KTP (i.e., 1\% (w/v) as opposed to 3\% (w/v)) could be hardly detected through the strongest peak at $1652 \mathrm{~cm}^{-1}$, attributed to the $\mathrm{C}=\mathrm{O}$ stretching. ${ }^{37}$ Unfortunately, FTIR spectra were not able to detect specific interactions in the dual drugloaded system due to the low intensity of the involved peaks.

Thermal Properties of Unloaded and Drug Loaded PLA Electrospun Scaffolds. Table II summarizes the main calorimetric data obtained from the heating run of unloaded and drug-loaded scaffolds from the two PLA grades, whereas the corresponding DSC traces are shown in Figure 5. Data 

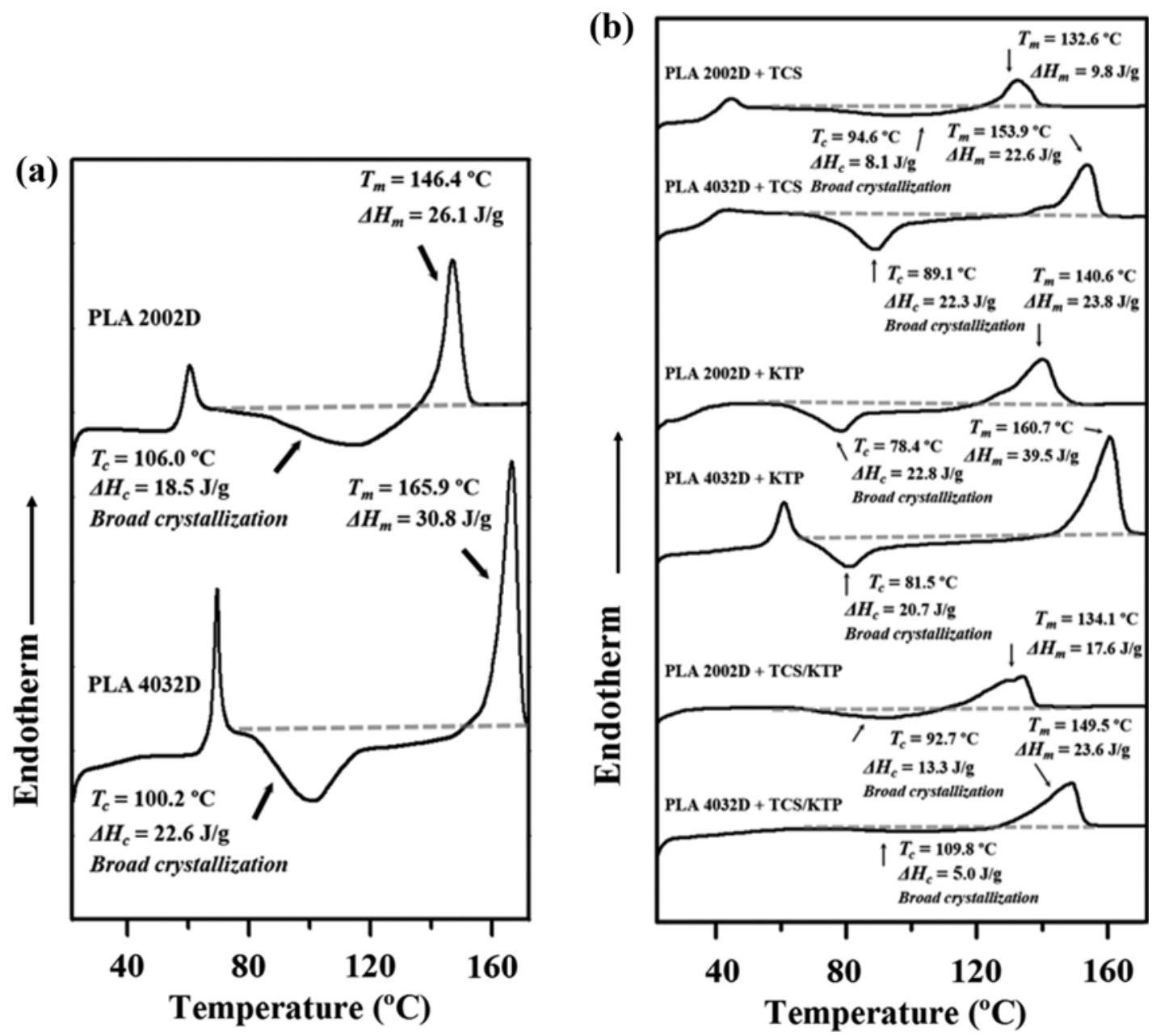

Figure 5. DSC heating runs $\left(20^{\circ} \mathrm{C} \cdot \mathrm{min}^{-1}\right)$ of unloaded (a) and drug-loaded (b) PLA 2002D and PLA 4032D electrospun scaffolds.

corresponding to the two PLA pellets are also given for comparative purposes. It is clear that both samples were manufactured in a semicrystalline form attained after annealing treatment. Values clearly reflect the impact of a small increase in D-lactide content (i.e., from $2 \% \mathrm{wt}$ to $4.2 \% \mathrm{wt}$ ) on both amorphous and crystalline phases, with remarkable changes in glass transition and melting temperatures, respectively. More interestingly, the thermal characteristics of electrospun samples are significantly different from the initial ones.

Both PLA 2002D and PLA 4032D electrospun samples became practically amorphous (degree of crystallinity, $C_{c}$, close to $0.07-0.08$, as indicated in Table II). Note however that cold crystallization peaks can be clearly observed during the heating run of both samples and relatively high degrees of crystallinity (i.e., 0.25 and 0.29 ) could be estimated from the subsequent melting enthalpies. These values were close to those deduced for the corresponding commercial pellet samples and clearly higher than those obtained from the heating run performed with commercial samples after being slowly cooled from the melt state. ${ }^{29}$ This feature suggests that electrospun PLA fibers can easily crystallize during a subsequent heating run as a consequence of a remarkable degree of molecular orientation that could be attained in the electrospinning process. The high relaxation peak observed for the PLA 4032D electrospun sample, which indicates the great ability of this PLA grade to achieve the equilibrium thermodynamic conditions characterized by higher stiffness and lower enthalpy and volume, is also worth noting. In addition, this close to equilibrium condition led to a fictive glass transition temperature $^{38}$ clearly lower than that observed in the initial sample of PLA $4032 \mathrm{D}$ (i.e., $62.4^{\circ} \mathrm{C}$ as opposed to $69.3{ }^{\circ} \mathrm{C}$ ) and close to those detected for both PLA 2002D initial and electrospun samples $\left(59.6{ }^{\circ} \mathrm{C}\right.$ and $\left.59.3{ }^{\circ} \mathrm{C}\right)$. Cold crystallization took place in a narrow temperature range for PLA 4032D, whereas the use of PLA 2002D involved a wide temperature range, suggesting greater difficulty for molecular rearrangement.

Incorporation of drugs had effects on thermal properties that depended on the polymer grade and the drug:

(a) Incorporation of TCS led to a significant decrease in the melting temperature of the two PLA samples (i.e., close to $13{ }^{\circ} \mathrm{C}$ ), suggesting partial incorporation into the crystalline 
Electrospun Scaffolds of Polylactide with a Different Enantiomeric Content and Loaded with Anti-Inflammatory and Antibacterial Drugs

Table II. Calorimetric Data from the Heating Scan Performed with Drug Loaded and Unloads in the Electrospun Samples

\begin{tabular}{ccccccccc}
\hline Sample & $T_{\mathrm{g}}\left({ }^{\circ} \mathrm{C}\right)$ & $\Delta C_{p}\left(\mathrm{~J} / \mathrm{g}{ }^{\circ} \mathrm{C}\right)$ & $T_{c}\left({ }^{\circ} \mathrm{C}\right)$ & $\Delta H_{c}^{b}(\mathrm{~J} / \mathrm{g})$ & $T_{m}{ }^{c}\left({ }^{\circ} \mathrm{C}\right)$ & $\Delta H_{m}{ }^{b}(\mathrm{~J} / \mathrm{g})$ & $\Delta H_{m}-\Delta H_{c}^{b}(\mathrm{~J} / \mathrm{g})$ & $\chi_{c}{ }^{d}$ \\
\hline PLA 2002D & 59.6 & 0.57 & - & - & 149.3 & 29.2 & 29.2 & 0.28 \\
PLA 2002D & 59.3 & 0.62 & 106.0 & 18.5 & 146.4 & 26.1 & 7.6 & $0.07,0.25$ \\
PLA 4032D ${ }^{a}$ & 69.3 & 0.23 & - & - & 167.0 & 35.0 & 35.0 & 0.33 \\
PLA 4032D & 62.4 & 0.55 & 100.2 & 22.6 & 165.9 & 30.8 & 8.2 & $0.08,0.29$ \\
PLA 2002D +TCS & 39.3 & 0.53 & 94.6 & 8.1 & 132.6 & 9.8 & 1.7 & $0.02,0.09$ \\
PLA 4032D +TCS & 40.1 & 0.50 & 89.1 & 22.3 & 153.9 & 22.6 & 0.3 & $<0.01,0.21$ \\
PLA 2002D + KTP & 43.7 & 0.40 & 78.4 & 22.8 & 140.6 & 23.8 & 1.0 & $0.01,0.22$ \\
PLA 4032 + KTP & 59.1 & 0.57 & 81.5 & 20.7 & 160.7 & 39.5 & 18.8 & $0.18,0.37$ \\
PLA 2002D + TCS/KTP & 51.6 & 0.47 & 92.7 & 13.3 & 134.1 & 17.6 & 4.3 & $0.04,0.17$ \\
PLA 4032D + TCS/KTP & 52.6 & 0.45 & 109.8 & 5.0 & 149.5 & 23.6 & 18.6 & $0.17,0.22$ \\
\hline
\end{tabular}

${ }^{a}$ Data obtained from commercial pellet samples. ${ }^{b}$ Values have been rescaled considering the theoretical content of TCS (i.e., 3\% (w/v)) and KTP (i.e., $1 \%(w / v))$. ${ }^{c}$ Temperature corresponding to the predominant melting peak. ${ }^{d}$ Determined by considering an estimated melting enthalpy of 106 $\mathrm{J} \cdot \mathrm{g}^{-1}$ for a $100 \%$ crystalline sample..$^{39,40}$ Left and right values correspond to crystallinities deduced for the as electrospun material (i.e., considering $\Delta H_{m}-\Delta H_{c}$ as the enthalpy associated to crystalline phase of the electrospun sample) and those attained after cold crystallization (i.e., considering $\Delta H_{m}$ as the enthalpy associated to the final crystalline phase), respectively.

structure. Both loaded samples were practically amorphous after electrospinning but the cold crystallization process was more favorable for the more regular PLA 4032D sample. Final crystallinities were logically lower than those of unloaded samples (i.e., 0.21 as opposed to 0.29 for PLA 4032D and 0.09 as opposed to 0.25 for PLA 2002D). TCS also had a plasticizing effect, as suggested by the significant decrease in the glass transition temperature. In addition, the trend to achieve the compact arrangement characteristic of an equilibrium condition was practically supressed for PLA 4032D derived scaffolds since the enthalpy relaxation peak was hardly detected.

(b) The melting point decrease was less significant (i.e., close to $6^{\circ} \mathrm{C}$ ) for scaffolds containing $\mathrm{KTP}$ as consequence of their lower drug load. In this case, the most significant feature was the high crystallinity attained after the cold crystallization process, which, in the case of PLA 4032D scaffolds, was even greater than for the unloaded sample. It seems that KTP could also act as a nucleating agent, as deduced from the decrease of the cold crystallization temperature and even from the narrower temperature range for the less crystalline PLA 2002D scaffold. Glass transition temperatures and enthalpy relaxation peaks decreased too, suggesting again a plasticizing effect of KTP and greater difficulty in obtaining the more compact equilibrium arrangement. Nevertheless, this trend was not completely supressed in the case of PLA 4032D.

(c) The dual drug-loaded samples showed peculiar DSC traces, which were the result of the effect caused by TCS and KTP. Thus, the decrease in the melting and glass transition temperatures was higher $\left(12-17^{\circ} \mathrm{C}\right.$ and $25-29^{\circ} \mathrm{C}$, respectively), enthalpy relaxation was completely suppressed and high crystallinity was attained before (for PLA 4032D sample) and after cold crystallization (for both PLA grades) due to the KTP nucleating effect. The high crystallinity that could again be directly attained in the electrospinning of the PLA 4032D grade in the presence of KTP is worth noting. Finally, melting peaks were, in this case, clearly complex due to the presence of crystals with different degrees of perfection (incorporation of impurities or/and different lamellar thicknesses).

Drug Release from Scaffolds of PLA with Different DLactide Content. A quantitative study was also attempted to follow the release of KTP and TCS from the two different PLA matrices. Release in the less hydrophilic medium containing ethanol (PBS:EtOH 3:7 v/v) was monitored through absorbance measurements at 270 and $281 \mathrm{~nm}$ for KTP and TCS, respectively, whereas values were measured at 260 and $290 \mathrm{~nm}$ for KTP and TCS, respectively, when hydrophilic PBS medium was employed. In all cases, concentrations were evaluated using the molar absorption coefficient $(\varepsilon)$ calculated for each drug in the corresponding release medium. These values were 7610 and $6908 \mathrm{M}^{-1} \mathrm{~cm}^{-1}$ for TCS in PBS: EtOH and PBS media, respectively, and 23,604 and 22,487 $\mathrm{M}^{-1} \mathrm{~cm}^{-1}$ for KTP in phosphate buffered saline:ethanol (PBS:EtOH) and PBS media, respectively.

Drug release plots in Figures 6 and 7 highlight differences depending on the PLA grade and logically on the selected medium. In all cases, the release attained a maximum value that mainly depends not only on the drug equilibrium distribution between microfibers and the release medium but also on the ability of microfibers to trap the drug efficiently. Hence, the final release percentage was always lower when the more hydrophilic PBS medium was used, in agreement with the hydrophobic character of the drugs and PLA. Furthermore, PLA 2002D microfibers showed the highest release percentages, probably as a consequence of the decrease in trapping efficiency caused by their lower molecular orientation and less dense molecular arrangement. Thus, for example, release 

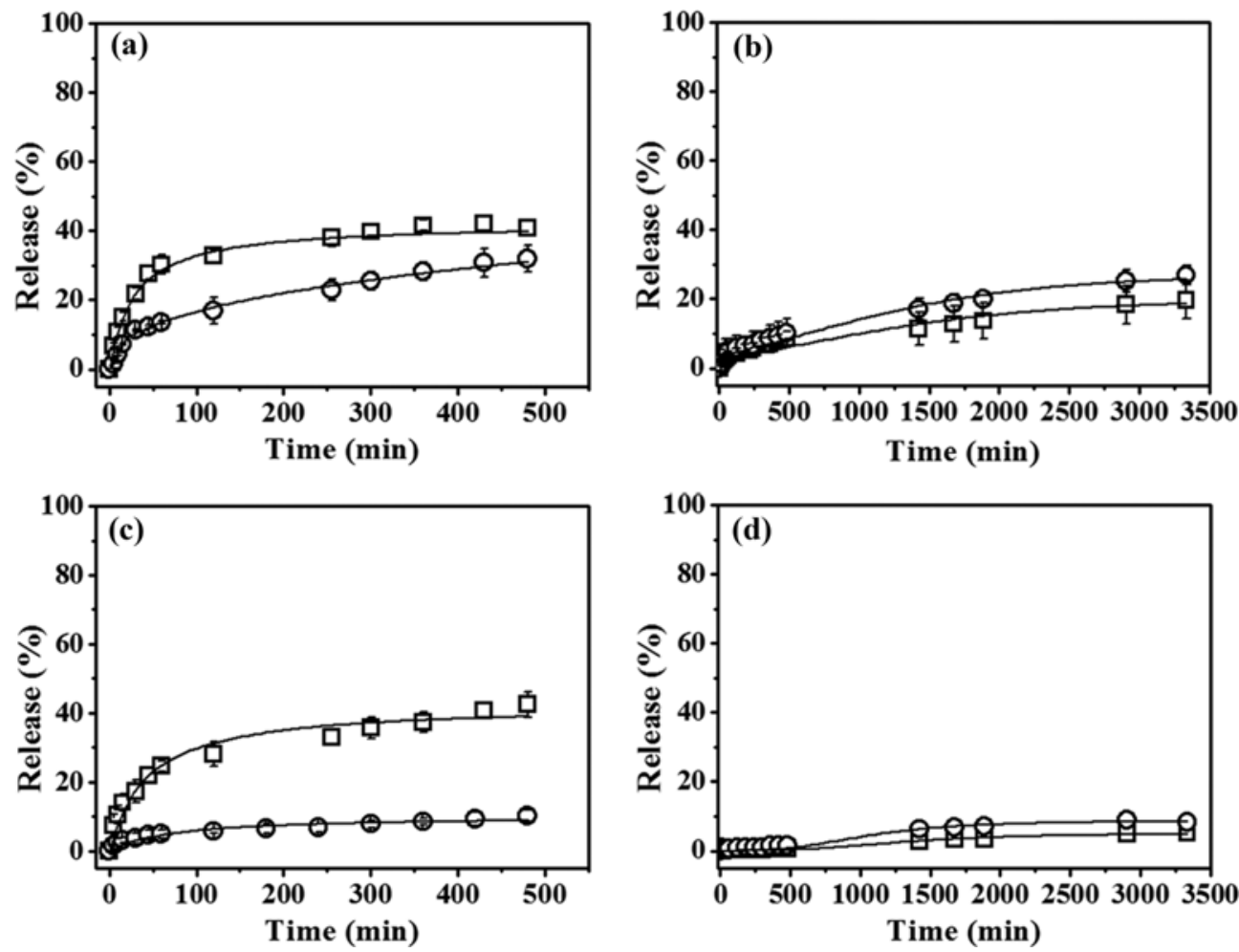

Figure 6. Release curves in PBS medium of TCS $(\square)$ and KTP $(\bigcirc)$ from single drug-loaded $(a, c)$ and dual drug-loaded (b,d) PLA 2002D $(\mathrm{a}, \mathrm{b})$ and PLA 4032D (c,d) electrospun scaffolds.
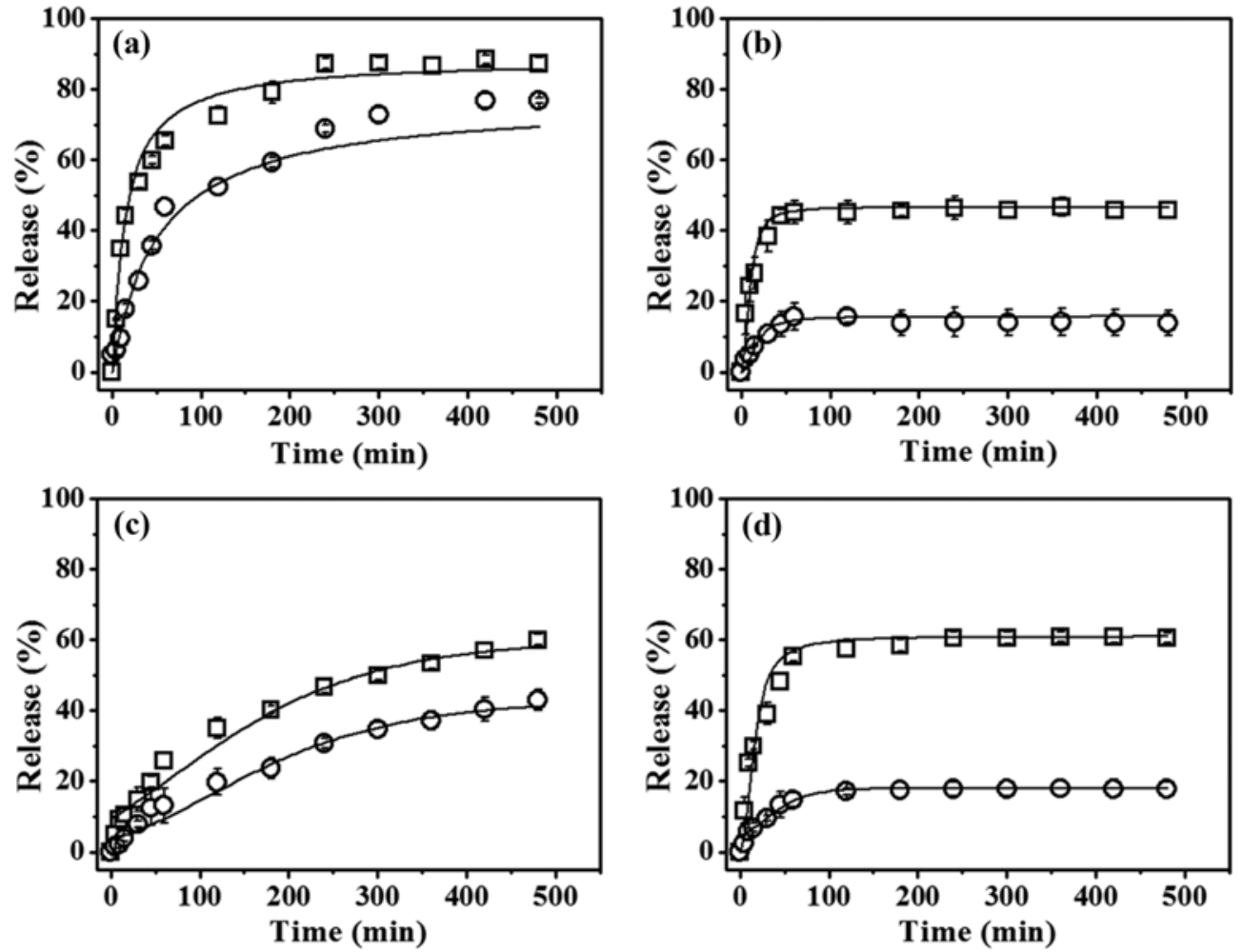

Figure 7. Release curves in PBS:EtOH medium of TCS $(\square)$ and KTP $(\bigcirc)$ from single drug-loaded (a,c) and dual drug-loaded (b,d) PLA 2002D (a,b) and PLA 4032D (c,d) electrospun scaffolds. 
of TCS and KTP from PLA 2002D scaffolds after $8 \mathrm{~h}$ of exposure to PBS medium rose to $40 \%$ and $30 \%$, respectively (Figure 6(a)), while these percentages decreased to 30\% and $5 \%$ for a similar exposure time when PLA 4032D scaffolds were employed (Figure 6(c)). A similar trend was observed in PBS:EtOH medium; specifically, the percentages of TCS and KTP release from PLA 2002D scaffolds after $8 \mathrm{~h}$ of exposure were, respectively, $80 \%$ and $60 \%$ (Figure 7(a)) and from PLA 4032D scaffolds, $50 \%$ and 30\%, respectively (Figure 7(c)).

The influence of release medium and type of polymer matrix on drug release was also observed when dual drugloaded scaffolds were evaluated (Figures 6(c), 6(d), 7(c), and 7(d)). An interesting observation about these dual systems is that the release profiles for each drug were different from those determined for single drug-loaded scaffolds. Specifically, a lower release percentage was always found in dual scaffolds. Note that fiber diameters were smaller for these dual systems, and consequently a contrary behavior should be expected if release were only controlled by molecular diffusion. Furthermore, drug release in dual systems seemed to depend on the physicochemical characteristics of the amorphous phase. Thus, decrease of the glass transition temperature or suppression of the aging effect may favour the release. It should be pointed out that crystallinity and molecular orientation were too similar in single and dual scaffolds loaded with KTP to justify the large differences found in the corresponding KTP release profiles. Therefore, TCS and KTP molecules should have some kind of intermolecular interactions inside microfibers that change equilibrium distribution or hinder molecular diffusion.
Plots in Figures 6 and 7 also show that release evolves in a gradual manner (i.e., without a significant burst effect indicative of the presence of drugs deposited on the microfiber surfaces only) until a significant value is attained.

In addition to the maximum achievable release, it is also interesting to compare the release rates, which can be easily determined from the experimental results. Several theoretical models have been postulated to fit experimental release profiles, typically first-order, ${ }^{41}$ Higuchi $^{42}$ and their combination. ${ }^{43}$ Release generally occurs in two different steps, i.e., a rapid release of molecules that should be deposited near on the surface of fibers and a slow release that should involve the diffusion of inner molecules through the polymer bulk towards the release medium. In this way, a combined model based on the Higuchi and first-order models (eqs. (1) and (2)) is usually used to describe the first $(0-60 \%)$ and second $(40-100 \%)$ parts of the release, respectively:

$$
\begin{array}{ll}
M_{t} / M_{0}=k_{H} t^{(1 / 2)} & \left(0 \leq M_{t} / M_{0} \leq 0.6\right) \\
\ln \left(1-M_{t} / M_{0}\right)=a-k_{1} t & \left(0.4 \leq M_{t} / M_{0} \leq 1.0\right)
\end{array}
$$

where $k_{H}$ is the Higuchi release constant, $k_{1}$ is the first-order release constant, $M_{t}$ is the percentage of drug released at time $t, M_{0}$ is the drug equilibrium percentage (considered as the maximum drug percentage) and $a$ is a constant.

Table III summarizes the values of $k_{H}$ and $k_{1}$ determined for representative scaffolds and release media (i.e., those with a significant release). Basically, the variations of $k_{1}$ reflect ability to reach the final equilibrium condition whereas $k_{H}$ values appear more interesting because they quantify and allow comparing drug release speed in the initial phase of delivery. This constant

\begin{tabular}{|c|c|c|c|c|}
\hline Sample & Drug & Medium & $k_{H}\left(\mathrm{~h}^{-0.5}\right)$ & $k_{1}\left(\mathrm{~h}^{-1}\right)$ \\
\hline PLA 2002D + TCS & TCS & PBS:EtOH & 0.78 & 0.58 \\
\hline PLA 4032D + TCS & TCS & PBS:EtOH & 0.35 & 0.38 \\
\hline PLA 2002D + TCS/KTP & TCS & PBS:EtOH & 0.78 & 0.97 \\
\hline PLA 4032D + TCS/KTP & TCS & PBS:EtOH & 0.79 & 1.10 \\
\hline PLA 2002D + TCS & TCS & PBS & 0.47 & 0.29 \\
\hline PLA 4032D + TCS & TCS & PBS & 0.48 & 1.48 \\
\hline PLA 2002D + TCS/KTP & TCS & PBS & 0.15 & 1.02 \\
\hline PLA 4032D + TCS/KTP & TCS & PBS & 0.04 & 5.61 \\
\hline PLA 2002D + KTP & KTP & PBS:EtOH & 0.50 & 0.39 \\
\hline PLA 4032D + KTP & KTP & PBS:EtOH & 0.35 & 0.40 \\
\hline PLA 2002D + TCS/KTP & KTP & PBS:EtOH & 0.25 & 1.60 \\
\hline PLA 4032D + TCS/KTP & KTP & PBS:EtOH & 0.24 & 1.65 \\
\hline PLA 2002D + KTP & KTP & PBS & 0.30 & 0.37 \\
\hline PLA 4032D + KTP & КTP & PBS & 0.10 & 0.30 \\
\hline PLA 2002D + TCS/KTP & KTP & PBS & 0.13 & 1.26 \\
\hline PLA 4032D + TCS/KTP & KTP & PBS & 0.06 & 2.24 \\
\hline
\end{tabular}

Table III. Kinetic Constants for the First (0-60\%) and Second Part (40-100\%) of the TCS and KTP Release 
is similar for TCS release in PBS:EtOH medium $(0.78-0.79$ $\mathrm{h}^{-0.5}$ ) from all scaffolds except from the single drug-loaded PLA 4032D system, where a decrease was detected $\left(0.35 \mathrm{~h}^{-0.5}\right)$. This constant clearly decreased $\left(0.04-0.48 \mathrm{~h}^{-0.5}\right)$ in PBS medium. Release of KTP proceeded at a slower rate than TCS in both media, with values of $0.24-0.50 \mathrm{~h}^{-0.5}$ and $0.06-0.30 \mathrm{~h}^{-0.5}$ for PBS:EtOH and PBS media, respectively. The lower rates for each range corresponded to scaffolds prepared from the more regular PLA 4032D matrix. Finally, it is worth mentioning that release of both KTP and TCS proceeded at a very slow rate ( 0.06 and $0.04 \mathrm{~h}^{-0.5}$, respectively) when dual drug-loaded systems and the more hydrophilic PBS medium were used.

Antibacterial Properties of TCS and KTP Loaded PLA Scaffolds. Antibacterial activity of PLA 2002D and PLA 4032D electrospun scaffolds loaded with TCS and its combination with KTP was tested against $M$. luteus and E. coli bacteria (as representative of Gram-positive and Gram-negative bacteria, respectively). It is well known that TCS is a broad-spectrum antiseptic with documented safety and efficacy against both types of bacteria. ${ }^{44,45}$ Basically, TCS blocks the active site of the enol-acyl carrier protein reductase enzyme, which is essential in the synthesis of fatty acids in bacteria. ${ }^{46}$

Bacterial growth curves for $E$. coli and M. luteus are shown
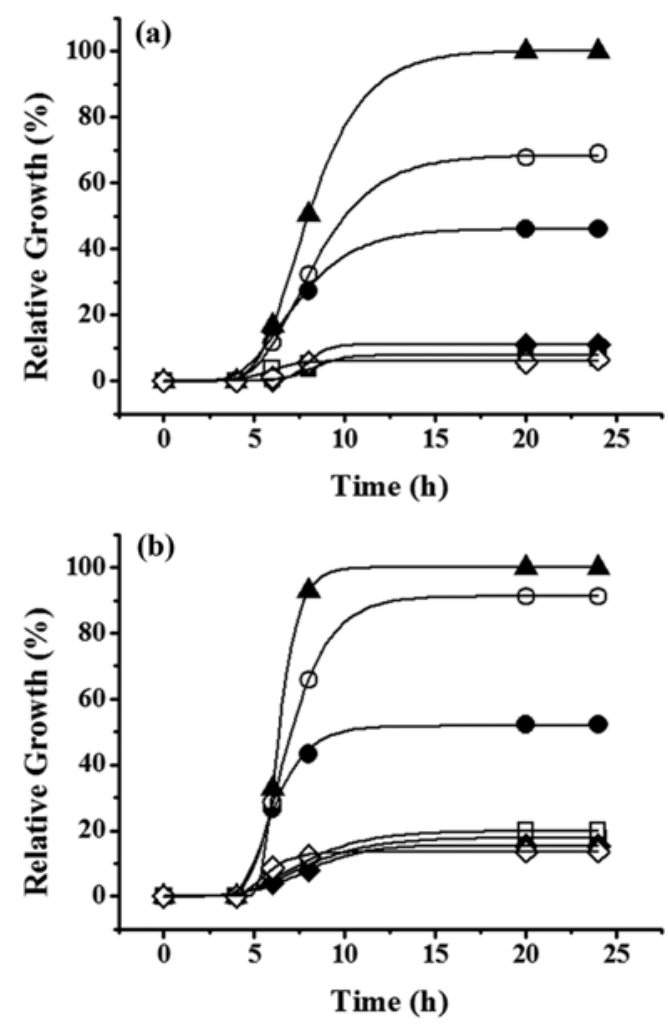

Figure 8. Relative growth of Escherichia coli (a) and Micrococcus luteus (b) on $\operatorname{TCS}(\boldsymbol{\square}, \square), \operatorname{KTP}(\boldsymbol{\bullet}, \bigcirc)$, and $\operatorname{TCS} / \operatorname{KTP}(\diamond, \diamond)$ loaded PLA electrospun scaffolds and polystyrene plate as postive control $(\boldsymbol{\Delta})$. Full and empty symbols correspond to PLA 2002D and PLA 4032D samples, respectively. in Figures 8(a) and (b), respectively. An initial lag phase (4-5 h) followed by an exponential phase associated with binary fission that stopped at growth levels clearly lower than for the positive control is observed. Therefore, for both types of bacteria, the results corroborate that single and dual TCS-loaded scaffolds were active materials for inhibition of bacterial growth. Bacterial growths between $5-10 \%$ and 10 $20 \%$ with respect to the positive control were observed for E. coli and M. luteus, respectively, after $24 \mathrm{~h}$ of culture.

Curves were essentially independent of the polymer matrix, and consequently antibacterial activity was not sensitive to the differences found in the release experiments. It seems that the high TCS content of scaffolds (i.e., $3 \%(\mathrm{w} / \mathrm{v}))$ ensured a sufficient release to inhibit bacterial growth even for matrices with a fairly low release. It is also important to indicate that the incorporation of KTP did not interfere with biological activity of TCS, at least at the assayed concentrations, and made possible the development of multifunctional materials with antibacterial and anti-inflammatory activities.

Interestingly, an intermediate level of inhibition of bacterial growth was also found for scaffolds only loaded with KTP, demonstrating antibacterial activity. Non-steroidal anti-inflammatory drugs (NSAID) are among the non-antibiotic drugs that have antimicrobial effect. Sodium diclofenac is probably the best NSAID archetype. Studies have demonstrated that this dug exhibits a broad activity against Gram-positive and Gramnegative bacteria for either in vitro or in vivo antimicrobial assays. The antimicrobial mechanism may be attributed to the inhibition of DNA synthesis in bacterial cells. ${ }^{47}$ In fact, KTP is a NSAID that inhibits cyclooxygenase, leading to analgesic, anti-inflammatory and antipyretic properties but at the same time it has some bactericide activity through a mechanism that remains unclear. ${ }^{48}$ Figure 8 clearly shows that the bactericide activity of KTP-loaded scaffolds depended on the selected PLA matrix; specifically, the greatest activity was observed for PLA 2002D. The result is in agreement with the lower crystallinity found for this sample and the higher release compared to the KTP-loaded PLA 4032D scaffold.

Quantification of bacterial adhesion is another checkpoint of bactericide activity. This activity is demonstrated for all TCS-loaded scaffolds since the percentage of attached bacteria was always statistically lower than that of positive controls, as shown in Figure 9. Nevertheless, activity was lower against $M$. luteus because Gram-positive bacteria are less sensitive to the effect of triclosan. Figure 9 also shows that single KTP-loaded scaffolds were unable to inhibit bacterial adhesion on their surface.

Cell Adhesion and Proliferation on Drug Loaded Scaffolds. Figures 10(a) and (b) show the behavior of two epithelial cell lines (MDCK and Vero) in terms of cell adhesion and proliferation on PLA electrospun scaffolds loaded with TCS and/or KTP. In general, scaffolds allowed adhesion of epithelial cells (Figure 10(a)), which is an important event for restarting epithelialization of a damaged tissue. Nevertheless, a 


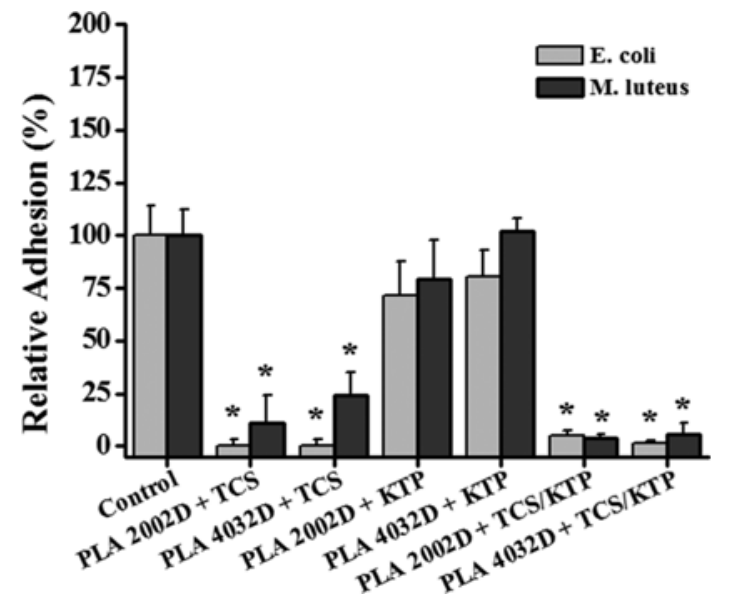

Figure 9. Escherichia coli (light gray) and Micrococcus luteus (dark gray) adhesion on polystyrene plate as positive control and TCS, KTP and TCS/KTP loaded electrospun scaffolds from PLA 2002D and PLA 4032D grades $(* p<0.05 v s$. control, Tukey test).
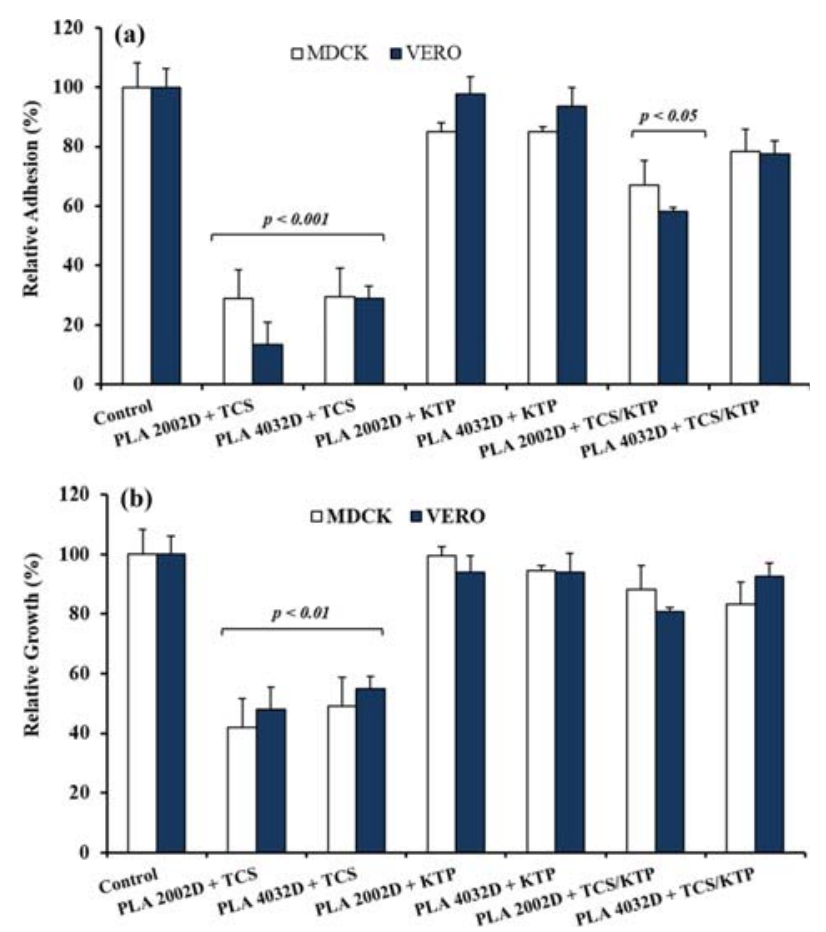

Figure 10. Adhesion (a) and proliferation (b) of MDCK (white) and Vero (grey) cells on polystyrene plate as positive control and TCS, KTP and TCS/KTP loaded electrospun scaffolds from PLA 2002D and PLA 4032D grades $(p<0.001, p<0.01$ and $p<0.05 v s$. control, ANOVA-Tukey test).

low percentage of cells (18-36\% with respect to the control) adhered to the loaded PLA scaffolds. This highly significant difference $(p<0.001)$ was due to the large percentage of loaded TCS (i.e., $3 \%(\mathrm{w} / \mathrm{v})$ ), which became cytotoxic to cells in culture. ${ }^{32}$ It is remarkable that a large percentage of adhesion was generally observed in dual drug-loaded scaffolds despite having the same TCS content. Only the PLA 2002D scaffold showed a slightly significant difference (borderline value with $p<0.048$ ). Cytotoxicity can therefore be modulated by incorporating KTP and even by modifying the PLA enantiomer composition. These results are in clear agreement with the release experiments, which indicated a decrease in the amount of delivered TCS when KTP was also incorporated into the microfibers and a higher release when the more amorphous PLA 2002D was employed as a matrix. Finally, a large percentage of cell adhesion was determined for PLA scaffolds loaded with the anti-inflammatory KTP, similarly to the positive control.

Adhered cells kept their proliferative activity and ultimately colonized the material, forming a monolayer tissue. Figure 10(b) quantifies cell growth, which was clearly related to the previous cell adhesion event. Thus, the percentage of cells growing on TCS-loaded PLA scaffolds was still lower than for the positive control (i.e., $42-55 \%$ ) but the difference was statistically less significant $(p<0.01)$ and relatively moderate. Again, the dual drug-loaded scaffold did not show any statistical difference with respect to the control, and therefore appears to be an appropriate $3 \mathrm{D}$ matrix for cell growth.

\section{Conclusions}

Continuous electrospun microfibers of polylactide containing antibacterial and anti-inflammatory agents (i.e., triclosan and ketoprofen) can be successfully prepared using optimized processing parameters and a mixture of chloroform, acetone and dimethylsulfoxide as solvent. Thermal properties of electrospun microfibers were highly dependent on small variations in the D-lactide content of PLA and the incorporation of small ratios of TCS and/or KTP. Specifically, microfibers containing KTP and prepared from the more regular PLA 4032D sample were crystalline as a result of the molecular orientation induced by the electrospinning process and probably by the nucleating effect of the incorporated drug.

Release of TCS and KTP from PLA electrospun scaffolds depended on the hydrophilicity of the media, the stereoregularity of the polymer matrix and the potential establishment of intermolecular interactions in dual drug-loaded scaffolds. Specifically, a decrease of the release percentage and the release rate for both drugs was detected in the binary system. This feature demonstrates the potential interest of the studied binary system since the intrinsic cytotoxicity of TCS could be supressed while the bactericide activity could be maintained.

Acknowledgments. Authors are in debt to supports from MINECO and FEDER (MAT2012-36205) and the Generalitat de Catalunya (2014SGR188).

\section{References}

(1) L. T. Lim, R. Auras, and M. Rubino, Prog. Polym. Sci., 33, 820 (2008). 
(2) M. Crank, M. Patel, F. Marscheider-Weidemann, J. Schleich, B. Hüsing, G. Angerer, and O. Wolf, Eds., in Techno-economic Feasibility of Large-scale Production of Bio-based Polymers in Europe, Technical Report EUR 22103 EN, European Commission, Joint Research Centre (DG JRC), Institute for Prospective Technological Studies, 2005.

(3) K. M. Nampoothiri, N. R. Nair and R. P. John, Bioresour. Technol., 101, 8493 (2010).

(4) R. Auras, B. Harte, and S. Selke, Macromol. Biosci, 4, 835 (2004).

(5) C. J. Weber, V. Haugaard, R. Festersen, and G. Bertelsen, Food Addit. Contam., 19, 172 (2002).

(6) J. J. Conn, R. Oyasu, M. Welsh, and J. M. Beal, Am. J. Surg., 128, 19 (1974).

(7) E. E. Schmitt and R. A. Polistina, Surgical Sutures, US Patent 3,297,033, USA (1967).

(8) C. Burger, K. Kabir, C. Rangger, M. Mueller, T. Minor, and R. Tolba, Arch. Orthop. Trauma Surg., 126, 695 (2006).

(9) B. Dhandayuthapani, Y. Yoshida, T. Maekawa, and D. S. Kumar, Int. J. Polym. Sci., 2011, 290602 (2011).

(10) J. Heller, Crit. Rev. Ther. Drug Carrier Syst., 1, 39 (1984).

(11) J. R. Sarasua, A. L. Arraiza, P. Balerdi, and I. Maiza, J. Mater. Sci., 40, 1855 (2005).

(12) J. K. Lee, K. H. Lee, and B. S. Jin, Eur. Polym. J., 37, 907 (2001).

(13) Z. W. Ma, M. Kotaki, R. Inai, and S. Ramakrishna. Tissue Eng., 11, 101 (2005).

(14) J. Zeng, X. Xu, X. Chen, Q. Liang, X. Bian, L. Yang, and X. Jing, J. Control. Release, 92, 227 (2003).

(15) W. J. Li, C. T. Laurencin, E. J. Catersonc, R. S. Tuan, and F. K. Ko, J. Biomed. Mater. Res., 60, 613 (2002).

(16) A. M. Piras, F. Chiellini, E. Chiellini, L. Nikkola, and N. Ashammakhi, J. Bioact. Compat. Polym., 23, 423 (2008).

(17) B. Song, C. T. Wu, and J. Chang, J. Biomed. Mater. Res. Part B: Appl. Biomater, 100B, 2178 (2012).

(18) Y. Wang, W. Qiao, B. Wang, Y. Zhang, P. Shao, and T. Yin, Polym. J., 43, 478 (2011).

(19) E. Llorens, M. M. Perez-Madrigal, E. Armelin, L. J. del Valle, J. Puiggali, and C. Aleman, RSC Adv., 4, 15245 (2014).

(20) T. Okuda, K. Tominaga, and S. Kidoaki, J. Control. Release, 143, 258 (2010).

(21) A. Schneider, X. Y. Wang, D. L. Kaplan, J. A. Garlick and C. Egles, Acta Biomater., 5, 2570 (2009).

(22) J. Cailloux, O. O. Santana, E. Franco-Urquiza, J. J. Bou, F. Carrasco, J. Gamez-Perez, and M. L. Maspoch, Express Polym. Lett., 7, 304 (2013).

(23) J. Cailloux, O. O. Santana, E. Franco-Urquiza, J. J. Bou, F.
Carrasco, and M. L. Maspoch, J. Mater. Sci., 49, 4093 (2014).

(24) R. Zurita, J. Puiggali, and A. Rodriguez-Galan, Macromol. Biosci., 6, 58 (2006).

(25) D. Li and Y. N. Xia, Adv. Mater., 16, 1151 (2004).

(26) S. R. Dhakate, B. Singla, M. Uppal, and R. B. Mathur, $A d v$ Mater. Lett., 1, 200 (2010).

(27) E. Luong-Van, L. Grondahl, K. N. Chua, K. W. Leong, V. Nurcombe, and S. M. Cool, Biomaterials, 27, 2042 (2006).

(28) G. Buschle-Diller, J. Cooper, J. Z. Xie, Y. Wu, J. Waldrup, and X. J. Ren, Cellulose, 14, 553 (2007).

(29) E. Llorens, L. J. del Valle, A. Diaz, M. T. Casas, and J. Puiggali, Macromol. Res., 21, 775 (2013).

(30) L. J. del Valle, R. Camps, A. Diaz, L. Franco, A. RodriguezGalan, and J. Puiggali, J. Polym. Res., 18, 1903 (2011).

(31) L. J. del Valle, A. Diaz, M. Royo, A. Rodriguez-Galan, and J. Puiggali, Express Polym. Lett., 6, 266 (2012).

(32) D. Karst and Y. Yang, J. Appl. Polym. Sci., 96, 416 (2005).

(33) D. M. Aragón, D. A. Chiappetta, J. Degrossi, E. F. Vargas, C. Bregni, A. Sosnik, and F. Martínez, Rev. Colomb. Cienc. Quim. Farm., 37, 241 (2008).

(34) J. Zeng, X. Chen, Q. Liang, X. Xu, and X. Jing, Macromol. Biosci., 4, 1118 (2004).

(35) H. Urayama, S. I. Moon, and Y. Kimura, Macromol. Mater. Eng., 288, 137 (2003).

(36) A. Celebioglu, O. C. O. Umu, T. Tekinay, and T. Uyar, Colloids Surf. B, 116, 612 (2014).

(37) M. L. Vueba, M. E. Pina, F. Veiga, J. J. Sousa, and L. A. E. B. de Carvalho, Int. J. Pharm., 307, 56 (2006).

(38) M. L. Cerrada and G. B. McKenna, Macromolecules, 33, 3065 (2000).

(39) T. Y. Cho and G. Strobl, Polymer, 47, 1036 (2006).

(40) J. R. Sarasua, R. E. Prud'homme, M. Wisniewski, A. Le Borgne, and N. Spassky, Macromolecules, 31, 3895 (1998).

(41) J. G. Wagner, J. Pharm. Sci., 58, 1253 (1969).

(42) T. Higuchi, J. Pharm. Sci., 52, 1145 (1963).

(43) R. Baker and R. W. Baker, Controlled Release of Biologically Active Agents, John Wiley \& Sons, New York, 1987.

(44) H. N. Bhargava and P. A. Leonard, Am. J. Infect. Control, 24, 209 (1996).

(45) L. M. McMurry, M. Oethinger, and S. B. Levy, Nature, 394, 531 (1998).

(46) C. W. Levy, A. Roujeinikova, S. Sedelnikova, A. R. Stuitje, A. R. Salabas, D. W. Rice, and J. B. Rafferty, Nature, 398, 383 (1999).

(47) S. G Dastidar, K. Ganguly, K. Chaudhuri, and A. N. Chakrabarty, Int. J. Antimicrob. Agents, 14, 249 (2000).

(48) H. A. Abbas, F. M. Serry, and E. M. El-Masry, Asian J. Res. Pharm. Sci., 2, 66 (2012). 